\section{Mit Hochfrequenz gegen Lungenmetastasen}

\begin{abstract}
Zur Behandlung von Lungenmetastasen werden oft minimal invasive Methoden wie die Hochfrequenz- oder Radiofrequenzablation (RFA) eingesetzt. Aber hat sie auch einen positiven Effekt auf das Überleben?
\end{abstract}

$\mathrm{M}$ inimal invasive Methoden wie die Hochfrequenzablation oder RFA werden als Alternative für Operationen bei der Behandlung von Lungenmetastasen eingesetzt. Sie ist beim Lungenkarzinom besonders effektiv, denn dieselbe Energiedosis führt in der Lunge aufgrund von Wärmeisolierung und niedriger elektrischer Leitfähigkeit des Gewebes - zu einem größeren Ablationsvolumen als in anderen Organen.

Aber wie wirkt sich dies auf Überleben und krankheitsfreies Überleben aus? In den Datenbanken von 2 Krebszentren wurden die Daten aller Patienten ausgewertet, die dort in einem Zeitraum von 8 Jahren wegen Lungenmetastasen behandelt worden waren. Die RFA erfolgte jeweils unter Anästhesie und mithilfe der Computertomografie (CT). $566 \mathrm{~Pa}$ tienten mit Primärtumoren in Kolon
(34\%), Rektum (18\%), Nieren (12\%), Weichteilgewebe (9\%) bzw. verschiedenen anderen Geweben (27\%) unterzogen sich insgesamt 642 RFA-Behandlungen für 1.037 Lungenmetastasen. $53 \%$ der Patienten hatten 1 Metastase, die übrigen bis zu 8 . In $75 \%$ der Fälle lagen diese unilateral vor, der mediane Durchmesser betrug $15 \mathrm{~mm}$. Extrapulmonale Metastasen, die einer lokalen Therapie zugänglich waren, kamen bei $22 \%$ der Patienten vor (49 Leber-, 16 Knochenund 60 andere Metastasen).

Das mediane Gesamtüberleben betrug 62 Monate. Nach 4 Jahren lag die lokale Kontrolle bei $89 \%$, die Krankheitskontrollrate in der Lunge bei 44,1\%. Primärer Krankheitsort, krankheitsfreies Intervall sowie Größe und Zahl von Metastasen waren in der multivariaten Analyse mit dem Gesamtüberleben assoziiert.
Die Progression am Ort der RFA verkürzte das Gesamtüberleben (Hazard Ratio [HR] 1,69; 95\%-Konfidenzintervall [95\%-KI] 1,13-2,54; $\mathrm{p}=0,011$ ). Bei den 293 Metastasen eines kolorektalen Karzinoms waren eine Größe von $>2 \mathrm{~cm}$ (HR $2,1 ; p=0,0027)$ und eine Zahl von $\geq 3 \mathrm{Me}$ tastasen (HR 1,86; $\mathrm{p}=0,011$ ) signifikant mit dem Gesamtüberleben assoziiert.

Mittels eines prognostischen Scores unterschied man anhand der 4 genannten prognostischen Faktoren 3 Patientengruppen: Diese 3 Gruppen zeigten 3-Jahres-Überlebensraten von 82,2, 69,5 und 53,6\% (p $\leq 0,0001)$; bei den Patienten mit kolorektalen Tumoren waren es $81,3,72,8$ und $57,9 \%(\mathrm{p}=0,005)$.

Fazit: Die RFA ist eine Therapieoption für Patienten mit kleinen Lungenmetastasen. Sie ist ebenso effektiv wie die chirurgische Entfernung der Metastasen, v. a. wenn deren Durchmesser zwischen 2 und $3 \mathrm{~cm}$ liegt.

Christina Berndt

Baère $T$ de et al. Radiofrequency ablation is a valid treatment option for lung metastases: Experience in 566 patients with 1037 metastases. Ann Oncol. 2015;26(5):987-91.

\section{Subgruppe gesucht für MUC1-spezifische Erhaltungstherapie beim NSCLC!}

\section{Das Glycoprotein MUC1 wird von vielen Tumorzellen mit veränderter Glykosylierung überexprimiert, so auch beim nichtkleinzelligen Lungen- karzinom (NSCLC). Das kann für eine spezifische Immuntherapie genutzt werden, über deren Nutzen aber noch keine Klarheit besteht.}

Ein in solches MUC1-Antigen-spezifisches Agens ist Tecemotid. In einer PhaseIII-Studie wurde jedoch der primäre Endpunkt - ein Überlebensvorteil der Erhaltungstherapie mit Tecemotid gegenüber Placebo nach gleichzeitiger oder sequenzieller Radio- und Chemotherapie - nicht erreicht. Das mediane Gesamtüberleben (OS) betrug unter Tecemotid 25,6, unter Placebo 22,3 Monate (adjustierte Hazard Ratio [HR] 0,88; $95 \%$-Konfidenzintervall [95\%-KI] 0,75-1,03; p = 0,123). An der Studie nahmen Patienten teil, die nach einer Erstlinien-Strahlenchemotherapie wegen eines nicht operablen NSCLC im Stadium III keinen Progress gezeigt hat- ten. Nun wurden aktualisierte Ergebnisse und solche zu bereits vorab definierten Subgruppen vorgestellt.

Auch nach einer etwa 20 Monate längeren Beobachtungszeit ergab sich kein Hinweis auf einen Überlebensvorteil durch die Immuntherapie. Das mediane OS lag bei 25,8 Monaten in der Tecemotid-Gruppe und bei 22,4 Monaten in der Placebogruppe (HR 0,89; $95 \%$-KI 0,771,03; $\mathrm{p}=0,111)$. Ein Überlebensvorteil zeigte sich aber bei Patienten, die zeitgleich eine Chemoradiotherapie erhalten hatten (OS: 29,4 vs. 20,8 Monate unter Placebo; HR 0,81, $95 \%$-KI 0,68-0,98; $\mathrm{p}=0,026)$. Dagegen war eine sequenzi- elle Chemoradiotherapie - erst Chemo-, dann Strahlentherapie - nicht mit einem Überlebensvorteil durch die Immuntherapie assoziiert.

Bei der Suche nach relevanten Biomarkern für ein Ansprechen zeigte sich für lösliches MUC1 und antinukleäre Antikörper (ANA) eine Korrelation zu einem verbesserten OS ( $\mathrm{p}$ für Interaktion $=0,0085$ bzw. 0,0022), nicht aber für die Lymphozytenzahl, das Verhältnis von Neutrophilen zu Lymphozyten oder die vordefinierten HLA-Typen.

Fazit: Die Überlebensdaten zeigen nach einer längeren Beobachtungszeit einen Hinweis auf einen Überlebensvorteil durch die Tecemotid-Therapie bei gleichzeitiger Chemo- und Strahlentherapie. Dabei könnten lösliches MUC1 und ANA als Biomarker für einen Therapieerfolg eine Rolle spielen. Friederike Klein

Mitchell $P$ et al. Tecemotide in unresectable stage III non-small-cell lung cancer in the phase III START study: updated overall survival and biomarker analyses. Ann Oncol. 2015;26(6):1134-42. 